\title{
Influencia de la motivación y del flow disposicional sobre la intención de realizar actividad físico-deportiva en adolescentes de cuatro países Influence of motivation and dispositional flow on the intention to dophysical activity in adolescents from fourcountries
}

*Evelia Franco Álvarez, *Javier Coterón López, **Valeria Gómez, ***Jorge Brito, ****Hernando Alejandro Martínez González

*Universidad Politécnica de Madrid (España), ${ }^{* *}$ Universidad de Flores (Argentina), ***Universidad de Cuenca (Ecuador), ${ }^{* * * *}$ Universidad del Quindío(Colombia)

Resumen. El objetivo de este estudio fue, en primer lugar, estudiar posibles diferencias en orientación motivacional, motivación intrínseca, flow disposicional e intención futura de ser físicamente activo en adolescentes de España, Argentina, Colombia y Ecuador; y en segundo lugar analizar el rol predictivo de las variables motivacionales mencionadas sobre la intención futura de ser físicamente activo. La muestra estuvo compuesta por 3990 estudiantes de centros educativos con edades comprendidas entre 12 y 18 años $(\mathrm{M}=14.55$; DT $=1.74)$ que respondieron diversos cuestionarios previamente validados y utilizados en español. Los resultados de los ANCOVAs realizados mostraron diferencias significativas entre los países analizados si bien el tamaño del efecto fue muy pequeño en todas ellas. En líneas generales, las similitudes fueron mayores entre los participantes de España y Argentina por un lado; y entre los de Colombia y Ecuador por otro. Los análisis de regresión por pasos revelaron que tanto la motivación intrínseca como el flow disposicional emergieron como principales predictores de la intención futura de los participantes de ser físicamente activos explicando entre un 34\% y un 52\% de la varianza. Los hallazgos del presente estudio sugieren la existencia de patrones motivacionales similares en adolescentes de diferentes países destacando el importante papel que adquiere el flow disposicional, independientemente del país, como predictor de la intención futura de realizar actividad físico-deportiva.

Palabras clave: motivación, flow, educación física, adolescentes, actividad física.

Abstract. The aims of this study were to analyze differences in motivational orientation, intrinsic motivation, dispositional flow and intention to be physically active among Argentine, Spanish, Colombian, and Ecuadorian adolescents; and to analyze the predictive role of the aforementioned motivational variables on the future intention to be physically active. The sample was composed by 3,990 students aged 12 to 18 ( $\mathrm{M}=14.55$, SD = 1.74) who completed questionnaires that were previously validated and used in Spanish contexts. Results of ANCOVAs showed significant differences between participants from the analyzed countries, although the effect size was very small in all of them. The greatest similarities were observed between Spanish and Argentinians, as well as between Colombians and Ecuadorians. Stepwise regression analyses showed that both intrinsic motivation and dispositional flow emerged as the main predictors of future intention to be physically active, as they both explained from $34 \%$ to $52 \%$ of the variance. The findings of the present study suggested the existence of similar motivational patterns among adolescents from different countries. Outcomes also highlighted the important role of dispositional flow in the prediction of future intentions to be physically active regardless of the country.

Keywords: motivation, flow, physical education, adolescents, physical activity.

\section{Introducción}

A pesar de los innegables beneficios que la práctica de actividad física (AF) tiene sobre las dimensiones fisiológica, psicológica y social de quienes la realizan (Carazo-Vargas y Moncada-Jiménez, 2015; Castillo y Moncada, 2012; Gomes et al., 2015), se observa en los últimos años un preocupante descenso de la misma en población adolescente que difícilmente alcanza los niveles recomendables para lograr beneficios sobre la salud física y social (Abarca-Sos, Zaragoza, Generelo y Julián, 2010; Oviedo et al., 2013).

La intención expresada por niños y adolescentes de practicar deporte o actividad física puede ser un buen indicador de su motivación hacia esta actividad, así como un fuerte predictor de su comportamiento futuro (Shephard y Trudeau, 2000) en base a lo expuesto por la teoría del comportamiento planificado (Ajzen, 1991). Desde esta perspectiva, el estudio de la asociación entre determinadas variables motivacionales y esta intención de práctica (e.g., Granero-Gallegos, et al., 2014) se ha desarrollado considerablemente en los últimos años desde las dos teorías motivacionales más relevantes: la teoría de las metas de logro (Ames, 1992; Nicholls, 1989) y la teoría de la autodeterminación (Deci y Ryan, 1985).

La teoría de las metas de logro estudia la conducta dirigida hacia una meta, incluidas las causas, dirección, y consecuencias de esta actividad, refiriéndose a cómo los individuos se acercan, se comprometen, y responden a actividades de logro, así como a las razones por las que se implican con ciertas conductas de ejecución. Según esta teoría, en los contextos de logro existen dos estados de implicación predominantes: una implicación al ego, en la que se percibe éxito cuando el rendimiento

Fecha recepción: 07-11-15. Fecha de aceptación: 07-06-16

Evelia Franco Álvarez

e.francoalvarez@gmail.com es superior al de los demás; y una implicación a la tarea, en la que el término meta significa mejora de la propia competencia personal. Este estado de implicación es el resultado de la interacción de factores disposicionales (orientación motivacional) y contextuales (clima motivacional).

Existe evidencia de que un estado de implicación a la tarea se relaciona con la intención de práctica futura de actividad física tanto directa (Moreno-González, 2014; Sproule, Wang, Morgan, McNeill, y McMorris, 2007) como indirectamente a través de la motivación intrínseca (Goudas, Biddle, y Fox, 1994a; Granero-Gallegos y BaenaExtremera, 2014). Aunque aparece menos frecuentemente en la literatura, algunos estudios también han encontrado una relación entre un estado de implicación al ego y la intención futura tanto directa (Lintunen, Valkonen, Leskinen, y Biddle, 1999) como indirectamente a través de la competencia percibida (Goudas, Biddle, y Fox, 1994b); y existe también evidencia de una relación negativa entre dicho estado de implicación y la participación en actividades físicas (Skjesol y Halvari, 2005). Por otra parte, se ha sugerido que la orientación motivacional al ego, si va acompañada de altos niveles de orientación motivacional a la tarea, también resulta positiva para la adherencia a la práctica de actividad física (Moreno-González, 2014).

La teoría de la autodeterminación analiza el grado en que las conductas humanas son autodeterminadas, es decir, el grado en que las personas realizan sus acciones al nivel más alto de reflexión y se comprometen en las mismas. Esta teoría general consta de 5 mini teorías: teoría de la evaluación cognitiva, teoría de la integración orgánica, teoría de las orientaciones de causalidad, teoría de las necesidades básicas y teoría de los contenidos de meta.

La mini teoría de la integración orgánica establece quela motivación es un continuo caracterizado por diferentes tipos de autodeterminación. De este modo, establece tres grandes niveles de motivación que son, de menor a mayor grado de autodeterminación: desmotivación, motivación extrínseca (con diferentes formas según el nivel de regulación) y 
motivación intrínseca. Esta última es, sin duda, el constructo que con más consistencia se ha visto asociado a comportamientos o actitudes relacionados con el desempeño de actividades físico-deportivas. Así, diversos estudios han establecido una relación entre la motivación intrínseca en clases de educación física y la intención de realizar actividad física durante su tiempo libre tanto directa (Baena-Extremera, GraneroGallegos, Sánchez-Fuentes, y Martínez-Molina, 2013; Belando, 2013; Pulido, Sánchez-Oliva, Amado, González-Ponce, y Sánchez-Miguel, 2014; Taylor, Ntoumanis, Standage, y Spray, 2010), como indirectamente a través de actitudes y percepciones de control hacia la actividad física (Belando, 2013; M. Hagger, Chatzisarantis, y Biddle, 2002).

A las dos perspectivas teóricas descritas, en los últimos años se ha añadido la investigación desde la teoría del flow. Csikszentmihalyi(1975) definió dicho estado como una experiencia óptima que implica una total absorción la tarea que se está realizando, un estado de concentración y una percepción alterada del tiempo. La experimentación de dicho estado positivo puede conllevar una mayor participación en actividades físicas debido a que los altos niveles de diversión y entretenimiento manifestados por quienes lo experimentan les hacen querer repetir de nuevo (Jackson, 1996; Kimiecik, 2000).

Tanto las orientaciones motivacionales como la motivación intrínseca y el flow disposicional han sido ampliamente estudiados en los últimos años, habiendo encontrado que altos niveles de orientación motivacional a la tarea (Christodoulidis, Papaioannou, y Digelidis, 2001), motivación intrínseca (Isorna, Rial, y Vaquero-Cristóbal, 2014) y flow disposicional (Cervelló, Moreno, Alonso, y Iglesias, 2006) se asocian a patrones adaptativos en lo que a práctica de actividad física se refiere.

La mayor parte de la investigación existente en este ámbito se ha realizado sobre muestras de un solo país. Si bien es cierto que la acumulación de evidencias en un mismo sentido permite comprobar tendencias comunes, Duda y Allison (1990) señalan que la investigación en el ámbito de la psicología de la actividad física y del deporte requiere la incorporación de análisis interculturales para evitar la propagación de teorías que pueden no ser generalizables debido a que la cultura y la etnia pueden ser factores que expliquen la variabilidad en los conocimientos, emociones y comportamientos de los individuos.

Existen trabajos que han considerado el estudio de variables motivacionales en diversos países (Ruiz-Juan y Baena-Extremera, 2013; Velázquez, Hernández, Garoz y Martínez, 2015; Vlachopoulos et al., 2013). A partir de la teoría de las metas de logro, diversos trabajos han comparado países de culturas occidentales y orientales, encontrando diferencias en cuanto a orientaciones motivacionales (e.g., Xiang, Lee, y Shen, 2001), concepciones de habilidad (Wang, Liu, Biddle, y Spray, 2005; Xiang etal., 2001), o motivación hacia la EF (Nishida, 1991; Ruiz, Graupera, Contreras, y Nishida, 2004). También se han encontrado diferencias entre adolescentes de países occidentales y orientales en las formas de motivación propuestas desde la teoría de la autodeterminación (Wang, Hagger, y Liu, 2009). La mayor parte de estos trabajos se han desarrollado comparando países sin una lengua materna común, no hallándose estudios que analicen el comportamiento de la motivación intrínseca y el flow en países que compartan un mismo idioma, encontrándose escasos estudios que analicen diferencias en variables motivacionales entre adolescentes de diferentes países (López-Walle, Tomás, Castillo, Tristán, y Balaguer, 2011; Ruiz-Juan y BaenaExtremera, 2015).

El análisis de las diferencias y similitudes en cuanto a componentes motivacionales en diferentes países parece conveniente de cara a poder generalizar tanto los supuestos de la teoría como los hallazgos fruto de investigaciones basadas en la misma.

En consecuencia, el objetivo del presente estudio fue analizar el comportamiento de las orientaciones motivacionales, motivación intrínseca, flow disposicional e intención de práctica futura en adolescentes españoles, argentinos, colombianos y ecuatorianos. En un segundo momento se pretendió analizar el papel predictivo que las variables motivacionales objeto de estudio podrían tener sobre la intención futura de ser físicamente activo. En base a estudios previos (e.g., Ruiz-Juan y Baena-Extremera) y al control que se llevó a cabo en el tratamiento estadísticos de otras variables que pudieran resultar influyentes (edad y nivel de actividad física realizada) se hipotetizó que se encontrarían patrones motivacionales semejantes entre los adolescentes de los diferentes países. En línea con lo encontrado en la literatura, también se hipotetizó que tanto la motivación intrínseca como el flow disposicional adoptarían un papel importante en la predicción de la intención futura de realizar actividad física, no pudiendo establecer una hipótesis en cuanto al rol de las orientaciones por la heterogeneidad de asociaciones entre estas variables halladas en estudios previos. Este estudio resulta pionero dada la escasez de trabajos que hayan analizado simultáneamente componentes motivacionales en cuatro países diferentes con una lengua común y por el tamaño de la muestra analizada.

\section{Método}

\section{Muestra}

La muestra del presente estudio estaba compuesta por un total de 3990 sujetos españoles, argentinos, colombianos y ecuatorianos de ambos géneros (2049 chicos y 1941 chicas), con edades comprendidas entre 12 y 18 años $(M=14.55$ años (DT =1.74). Para la selección de la misma se siguió un método no probabilístico intencional o de conveniencia.

En cuanto a la distribución por edades, la muestra estaba compuesta por 574 participantes de 12 años, 709 participantes de 13 años, 743 participantes de 14 años, 655 participantes de 15 años, 691 participantes de 16 años, 439 participantes de 17 años y 170 participantes de 18 años.

En la Tabla 1 se muestra la distribución de la muestra por países así como los descriptivos estadísticos de la edad y el nivel de actividad física realizado, calculado en unidades de medida metabólica(METs) de cada país.

Tabla 1

Distribución de la muestra por país y género, y estadísticos descriptivos de la edad y de la actividad física medida en unidades de medida del índice metabólico (METs) en cada país

\begin{tabular}{cccccc}
\multicolumn{6}{c}{ actividad física medida en unidades de medida del índice metabólico (METs) en cada país } \\
\hline & \multirow{2}{*}{ total } & \multirow{2}{*}{ Hombres } & Mujeres & Edad & METs \\
& & & & MT) & M (DT) \\
\hline España & 930 & 445 & 485 & $14.16(1.56)$ & $55.71(25.55)$ \\
Argentina & 1323 & 660 & 663 & $15.07(1.77)$ & $46.27(26.06)$ \\
Colombia & 1095 & 515 & 580 & $14.76(1.61)$ & $56.11(27.27)$ \\
Ecuador & 641 & 429 & 212 & $13.72(1.67)$ & $55.62(23.25)$ \\
\hline
\end{tabular}

\section{Instrumentos}

A continuación se describen cada una de las escalas utilizadas en la presenteinvestigación.

Orientación motivacional. Se utilizó la versión española (Peiró y Sanchis, 2004) del Task and Ego Orientation Scale Questionnaire (TEOSQ; Walling y Duda, 1995). Este instrumento consta de 16 ítems, midiendo la orientación a la tarea (8 ítems; e.g., «cuando aprendo una nueva habilidad») y la orientación al ego (8 ítems; e.g., «cuando soy el/ la único/a capaz de realizar un ejercicio»). El cuestionario va precedido de la frase «Yo siento que tengo más éxito en las clases de educación física cuando...» Las respuestas a dicho cuestionario son cerradas y se responden con una escala tipo Likert cuya puntuación oscila entre 1= totalmente en desacuerdo y $5=$ totalmente de acuerdo. Dicho cuestionario mostró valores alpha de .82 para el factor orientación a la tarea, y de .87 para el factor orientación al ego.

Flow disposicional. Se utilizo la versión española adaptada a la educación física (González-Cutre, 2009) de la Dispositional Flow Scale2 (DFS-2) de Jackson y Eklund (2002) para medir la disposición de los alumnos a experimentar un estado de flow durante las clases de educación física. Esta herramienta mide la disposición a experimentar el estado de flow a partir de nueve factores. A continuación se presentan dichos factores junto al valor alpha correspondiente a cada uno: equilibrio reto-habilidad (.73), automatismo (.72), claridad de objetivos (.76), claridad en el feedback (.73), concentración (.75), sentimiento de control (.76), pérdida de la auto-conciencia (.79), distorsión del tiempo (.70) y experiencia autotélica (.80). Está compuesta por 36 ítems (cuatro ítems para cada uno de los nueve factores motivacionales mencionados; e.g., «sabía que mi capacidad me permitiría hacer frente al desafío que se me planteaba»). En el cuestionario se le pregunta a los estudiantes sobre su 
experiencia en la clase de educación física. Las respuestas a dicho cuestionario son cerradas y se responden con una escala tipo Líkert cuya puntuación oscila entre $1=$ totalmente en desacuerdo y $5=$ totalmente de acuerdo.

Motivación intrínseca. Se utilizaron los ítems correspondientes al factor motivación intrínseca de la versión española (Moreno, GonzálezCutre, y Chillón, 2009) del Perceived Locus of Causality Scale (PLOC; Goudas, et al., 1994b). La PLOC está compuesta por 20 ítems, cuatro midiendo cada una de las dimensiones; desmotivación (e.g., «pero no sé realmente por qué), la regulación externa (e.g., «porque tendré problemas si no lo hago»), la regulación introyectada (e.g., «porque me preocupa cuando no lo hago»), identificada (e.g., «porque quiero aprender habilidades deportivas») y la motivación intrínseca (e.g., «porque la educación física es divertida) en la clase de educación física El cuestionario va precedido por la frase «Participo en esta clase de educación física...» Las respuestas a dicho cuestionario fueron cerradas y se respondieron con una escala tipo Líkert cuya puntuación oscila entre $1=$ totalmente en desacuerdo y $5=$ totalmente de acuerdo. El factor motivación intrínseca mostró un valor alpha de .87.

Intención futura de ser físicamente activo. Se utilizó la Intention to be Physically Active Scale (IPAS; Hein, Müür, y Koka, 2004) en su versión española (Moreno, Moreno, y Cervelló, 2007). Esta escala se compone de cinco ítems (e.g., «después de terminar el instituto, me gustaría mantenerme físicamente activo/a») para medir la intención del sujeto de ser físicamente activo tras su paso por las diversas instituciones educativas. El cuestionario va precedido por la frase «Respecto a tu intención de practicar alguna actividad físico-deportiva...». Las respuestas a dicho cuestionario son cerradas y se responden con una escala tipo Líkert cuya puntuación oscila entre 1= totalmente en desacuerdo y 5 = totalmente de acuerdo. La escala mostró un valor alpha de .79.

Práctica de actividad física actual. Se utilizó el Leisure-TimeExercise Questionnaire (G-LTEQ; Godin y Shephard, 1985) traducida al español. La herramienta consta de dos partes. En la primera, se le pregunta a los participantes cuántas veces a la semana realizan actividad suave, moderada, o vigorosa. A partir de las respuestas se obtiene una puntuación total $(9 \mathrm{x}$ veces que realizan actividad vigorosa $+5 \mathrm{x}$ veces que realizan actividad moderada $+3 \mathrm{x}$ veces que realizan actividad suave) que se interpreta como las unidades de equivalencia metabólica (METs) semanales.

\section{Procedimiento}

Se estableció contacto con los equipos directivos y/o responsables del departamento de educación física de los diferentes centros de enseñanza para informarles de los objetivos y pedirles su colaboración. Tras la obtención de los permisos pertinentes conforme las indicaciones de los principios éticos y códigos de conducta de laAmerican Psychological Association (2002) para este tipo de investigaciones, la administración de los cuestionarios fue llevada a cabo en cada país por un miembro del equipo investigador, entre marzo y diciembre de 2014. Los cuestionarios se administraron durante una clase de educación física en ausencia del profesor, dando las instrucciones necesarias para la cumplimentación e insistiendo en el anonimato y sinceridad en las respuestas. La participación fue voluntaria y se respetaron todos los procedimientos éticos de recogida de datos. El tiempo requerido para rellenar los cuestionarios fue de aproximadamente 25 minutos, variando ligeramente según la edad del estudiante.

\section{Análisis de datos}

En primer lugar se calcularon los estadísticos descriptivos de las diferentes variables de estudio, segmentando la muestra según el país al que pertenecían los sujetos.

A continuación se realizó un análisis multivariante de la covarianza (MANCOVA) para detectar posibles diferencias en las variables de estudio (orientación motivacional, flow disposicional, motivación intrínseca, intención futura de ser físicamente activo) en función del país controlando el efecto que pudieran tener la edad y el nivel actual de actividad física realizado. Una vez detectado un efecto multivariante significativo se procedió a realizar los análisis univariantes de covarianza (ANCOVAs) y el test de Bonferroni para los análisis a posteriori.

Por último, se llevó a cabo un análisis por regresión por pasos en cada uno de los países utilizando la intención futura de ser físicamente activo como variable dependiente.

Se utilizó el paquete estadístico SPSS 20.0.

\section{Resultados}

La Tabla 2 muestra los estadísticos descriptivos de las muestras de los cuatro países en las variables de estudio.

Tabla 2

Descriptivos estadísticos de las variables dependientes objeto de estudio según el factor país España (n=930) Argentina (n=1323) Colombia ( $\mathrm{n}=1096)$ Ecuador ( $\mathrm{n}=641)$

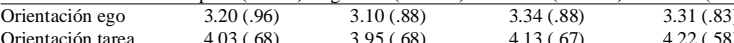
$\begin{array}{lllll}\text { Orientación tarea } & 4.03(.68) & 3.95(.68) & 4.13(.67) & 4.22(.58) \\ \text { Flow disposicional } & 3.54(67) & 3.54(.60) & 3.74(65) & 3.71(.59)\end{array}$ \begin{tabular}{lllll} 
How disposicional & $3.54(.67)$ & $3.54(.60)$ & $3.74(.65)$ & $3.71(.59)$ \\
\hline
\end{tabular} \begin{tabular}{lllll} 
Motivación intrinseca & $3.86(.98)$ & $3.75(.99)$ & $3.92(1.01)$ & $4.04(.88)$ \\
Intención futura AF & $4.01(.86)$ & $3.86(.87)$ & $4.03(.88)$ & $4.13(.76)$ \\
\hline
\end{tabular}

El análisis de la distribución de estas variables confirmó que podían ser consideradas normales y, por tanto, susceptibles de ser estudiadas a partir de pruebas paramétricas.

El efecto de la variable independiente país sobre las variables dependientes objeto de estudio se determinó a partir de un análisis multivariado de la covarianza (MANCOVA) utilizando el país como factor tras ajustar posibles diferencias debidas a la edad y al nivel de actividad física de los participantes. Los resultados mostraron un efecto multivariante significativo (ë de Wilks $(15,10926)=10.35, p<.01$. A continuación, se realizaron análisis univariados (ANCOVAs) en cada variable dependiente controlando los efectos de la edad y el nivel de actividad física.

En la Tabla 3 se muestran los resultados de los análisis univariados presentando los valores de las medias ajustadas tras controlar el efecto de las covariables. Los resultados revelaron un efecto significativo del país sobre las cinco variables dependientes. El tamaño del efecto, de acuerdo al criterio de Cohen (1988) fue muy pequeño. También se indican los resultados de los análisis a posteriori realizados con el test de Bonferroni

En un segundo momento, como se expone en la Tabla 4, el análisis

Tabla 3

Resultados de los ANCOVAs: medias ajustadas (controlando la edad y el nivel de actividad física), \begin{tabular}{ccccccccc} 
valor de $F$, valor de significación y & tamaño del efecto en las variables dependientes \\
\hline Es & $\mathrm{Ar}$ & $\mathrm{Co}$ & $\mathrm{Ec}$ & $\mathrm{F}$ & $\mathrm{p}$ & $\mathrm{n}^{2}$ & & Diferencias
\end{tabular}

\begin{tabular}{lcccccccc} 
& $(\mathrm{n}=930)$ & $(\mathrm{n}=1323)$ & $(\mathrm{n}=1096)$ & $(\mathrm{n}=641)$ & $\mathrm{F}$ & $\mathrm{p}$ & $\mathrm{n}^{2}$ & \\
\hline Orientación & 3.18 & 3.14 & 3.33 & 3.28 & $10.47<.05$ & .001 & Co.> Es., Ar., Ec., Ec.> Ar. \\
ego & $(.03)$ & $(.03)$ & $(.03)$ & $(.04)$ & & & &
\end{tabular}

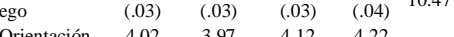

$\begin{array}{llllllll}\text { Orientación } & 4.02 & 3.97 & 4.12 & 4.22 & 21.87<.05 & .016 & \text { Co. y Ec. }>\text { Es. y Ar. } \\ \text { tarea } & (.02) & (.02) & (.02) & (.03) & \end{array}$

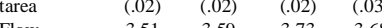

$\begin{array}{lllllll}\text { Flow } & 3.51 & 3.59 & 3.73 & 3.68 & 23.73<.05 .018 & \text { Co. y Ec. }>\text { Ar. }>\text { Es. } \\ \text { disposicional } & (.02) & (.02) & (.02) & (.03) & \end{array}$

disposicional $\left(\begin{array}{llll}.02) & (.02) & (.02) & (.03)\end{array}\right.$

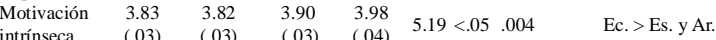

\begin{tabular}{lllll} 
intrínseca & $(.03)$ & $(.03)$ & $(.03)$ & $(.04)$ \\
Intención & 3.97 & 3.95 & 3.99 & 4.07 \\
fura & $(.03)$ & $(.02)$ & $(.03)$ & $(.03)$ \\
\hline
\end{tabular}

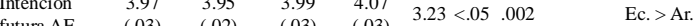

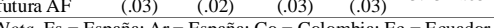

Tabla 4

Análisis de regresión lineal por pasos de la predicción de la intención de ser físicamente activo en

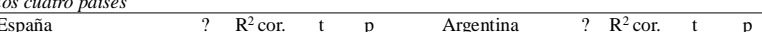

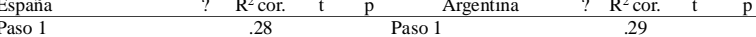
$\begin{array}{llllllllll}\text { Flow disposicional } & .68 & & 19.23 .001 & \text { Flow disposicional } & .79 & 23.43 & .001\end{array}$

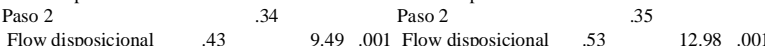

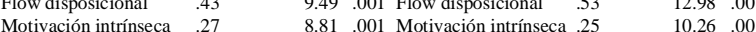

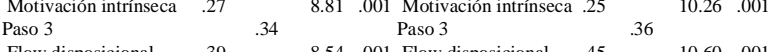

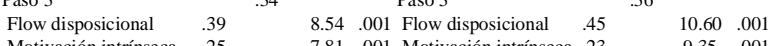
$\begin{array}{lllllll}\text { Motivación intrínseca } & .25 & 7.81 & .001 \text { Motivación intrínseca } & .23 & 9.35 & .001\end{array}$ $\begin{array}{lllllllll}\text { Orientación tarea } & .11 & & 2.88 & .004 & \text { Orientación tarea } & .19 & 5.94 & .001\end{array}$

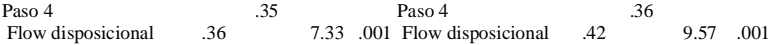

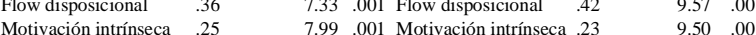

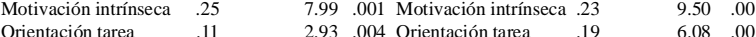

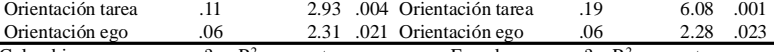

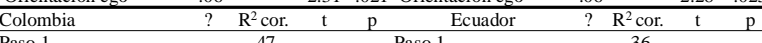

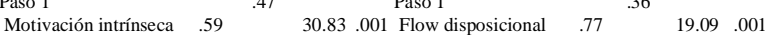

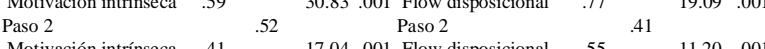

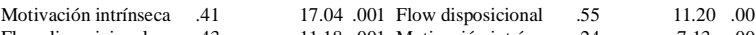
$\begin{array}{lllllllll}\text { Flow disposicional } & 43 & & 11.18 .001 & \text { Motivación intrínseca } & .24 & 7.13 & .001\end{array}$ $\begin{array}{llllllllll}\text { Flow disposicional } & .39 & .53 & 16.12 .001 \text { Flow disposicional } & .51 & & .41 & 9.39 & .001\end{array}$

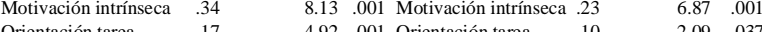


de regresión por pasos mostró que todas las variables resultaron significativas en las muestras española y argentina, no emergiendo el ego como predictor de la intención futura de ser físicamente activo en las muestras colombiana y ecuatoriana. En losmodelos resultantes la varianza explicada osciló entre el 35\% en España y el 53\% en Colombia siendo en todos los casos las variables de flow disposicional y motivación intrínseca las que mayor proporción de varianza de la intención futura de realizar actividad físico-deportiva explicaron.

\section{Discusión}

El primer objetivo de este estudio fue el análisis de las variables orientación motivacional, motivación intrínseca, flow disposicional e intención futura de ser físicamente activo en adolescentes de España, Argentina, Colombia y Ecuador.

El MANCOVA y los subsiguientes ANCOVAs realizados mostraron que existían diferencias en todas las variables en función del país, siendo en líneas generales los resultados de la muestra argentina similar a los de la muestra española en las variables motivacionales y el flow disposicional, situándose los niveles de ambas muestras por debajo de los sujetos colombianos y ecuatorianos quienes, a su vez, también mostraron similitudes entre sí. En la intención de práctica de actividad física en el futuro los jóvenes argentinos difieren del resto mostrando puntuaciones inferiores.

A pesar de la detección de estas diferencias al observar las medias obtenidas en las diferentes variables vemos cómo no existen grandes desigualdades entre los diversos países. Esta realidad puede explicarse porque el tipo de prueba estadística aplicada es bastante sensible a tamaño de la muestra y puede ocurrir que en muestras tan grandes como la utilizada en el presente estudio (3990 sujetos) incluso con un efecto pequeño, el estadístico resulte significativo(Quezada, 2007). Prueba de ello es el tamaño del efecto observado en los diferentes ANCOVAs, en todos los casos por debajo del 0.2 establecido por Cohen (1988) como una magnitud del efecto pequeña.

Existen pocas referencias de estudios que hayan comparado poblaciones de distintos países latinoamericanos. En su tesis doctoral GarcíaSandoval (2013) analiza una muestra de jóvenes mejicanos y españoles y se plantea, entre otros objetivos, identificar y diferenciar los motivos más importantes que llevan a los participantes a practicar actividad física, las barreras para la práctica físico-deportiva, y el nivel de ejercicio físico. En líneas generales no se hallan grandes diferencias entre jóvenes mejicanos y españoles en las variables estudiadas. El motivo más importante para la realización de actividad física en ambas muestras es la prevención y la salud positiva, mientras que la principal barrera para la práctica de ejercicio son las obligaciones escolares y la falta de tiempo. Entre las diferencias encontradas podemos mencionar que la barrera de la imagen corporal y la ansiedad física parece más importante entre jóvenes españoles que mejicanos, y que la muestra de estudiantes españoles reportó percibirse más activa que la muestra de estudiantes mejicanos. Ruiz-Juan y Baena-Extremera (2015), en un estudio con una muestra de participantes españoles, costarricenses y mejicanos de edades similares a las del presente estudio, analizaron la predicción de las orientaciones de meta en EF a partir de la satisfacción, la motivación y las creencias de éxito. En dicho estudio los resultados mostraron tendencias similares en los tres países. En otro trabajo realizado con estudiantes de EF con edades similares en España y Brasil (Velázquez et al., 2015) se analizó la relación entre actividad física, la percepción de autoeficacia motriz y la satisfacción con la clase y el profesorado de EF detectándose un papel predictivo similar de las variables en las muestras de ambos países.

Si bien resultaron significativas las diferencias entre los diferentes grupos del presente estudio, el ínfimo valor del tamaño del efecto sugiere la existencia de similitudes entre los patrones motivacionales en línea con lo encontrado en la literatura.

El segundo objetivo del presente trabajo fue analizar el rol predictivo de las orientaciones motivacionales, la motivación intrínseca y el flow disposicional sobre la intención futura de ser físicamente activo.
Tal y como se hipotetizaba, tanto la motivación intrínseca como el flow motivacional emergieron como principales predictores de la intención futura de los adolescentes de ser físicamente activos.

El análisis de regresión por pasos reveló que el flow disposicional fue el primer predictor de la variable dependiente en España, Argentina y Ecuador, mientras que la motivación intrínseca lo fue en Colombia. Independientemente del orden en que aparecieran en el modelo, lo cierto es que en todas las muestras analizadas resultó relevante la proporción de varianza explicada de la variable dependiente a través de estas dos variables.

La literatura es prolija en estudios que han hallado asociaciones entre la motivación intrínseca y la intención futura de ser físicamente activo variables (e.g., Hein et al., 2004; Taylor et al., 2010) siendo sin embargo escasos los estudios que señalan la relación entre esta última y la disposición a experimentar el flow.

El papel predictivo del flow disposicional en la intención de ser físicamente activo en el futuro independientemente del país sugiere el importante rol que adquiere esta variable en la explicación de diferencias en la participación de actividades físico-deportivas. Tal y como se apunta desde el enfoque fenomenológico del flow (Csikszentmihalyi, Abuhamdeh y Nakamura, 2005) los resultados sugieren la importancia que las sensaciones que los estudiantes experimenten en la clase de EF tendrán sobre sus actitudes hacia la práctica deAF. Este hallazgo está en línea con lo encontrado en trabajos anteriores que sugieren el rol predictor que adquiere el flow sobre variables adaptativas en lo que respecta a la adherencia a la práctica de actividad físico-deportiva tales como la satisfacción con la actividad realizada (Jackson y Marsh, 1996), el compromiso con la misma(Deci y Ryan, 1985; Karageorghis, Vlachopoulos, \& Terry, 2000), y la aparición de emociones positivas tras la realización de la actividad como la revitalización o la tranquilidad (Karageorghis, et al., 2000).

Cabe señalar que el presente estudio presenta algunas limitaciones como las derivadas del uso de una herramienta para medir actividad física basada en la autorrespuesta (Bauman y Merom, 2002). Además, la naturaleza correlacional del trabajo no permite inferir causalidad de las relaciones encontradas en el trabajo. No obstante, este estudio ha supuesto un pequeño paso más en la comprensión del comportamiento y relaciones entre variables motivacionales en diferentes países. Son necesarias más investigaciones que analicen la influencia de las variables de este estudio sobre aspectos relacionados con el aprendizaje y adquisición de habilidades a partir de diferentes diseños (longitudinales y cuasiexperimentales).

\section{Conclusiones}

El presente estudio se suma a la literatura existente relativa al análisis de variables motivacionales con la adherencia a la práctica de actividad física, explorando el comportamiento de algunas variables relevantes en adolescentes de distintos países. Los hallazgos sugieren que las diferencias encontradas entre adolescentes de distintos países no son relevantes detectándose patrones motivacionales similares entre los mismos. El papel protagonista del flow disposicional como predictor de la intención futura de ser físicamente activo sugiere la necesidad de diseñar y/o poner en práctica estrategias para mejorar la disposición a experimentar dicho estado (Mandigo y Thompson, 1998) en contextos de EF de diferentes países.

Tal y como señalan los autores mencionados, y en línea con estudios previos, contextos que favorezcan un contexto estructurado que promueva la autonomía del alumnado, estrategias para favorecer la diversión durante la clase o la claridad en la presentación de objetivos y de feedback parecen tener un efecto positivo sobre la generación de patrones motivacionales adaptativos.

\section{Agradecimientos}

La realización de este trabajo fue posible gracias a los proyecto de investigación «Estudio de la influencia del clima motivacional sobre la 
motivación intrínseca y la intención de ser físicamente activo» (Ref. AL14-PID-40), financiado por la Universidad Politécnica de Madrid (España); y «Análisis de los perfiles motivacionales del estudiante de Educación Física en Secundariay su relación con la intención de práctica futura» (R69-CSUFLO) financiado por la Universidad de Flores (Argentina).

\section{Referencias}

Abarca-Sos, A., Zaragoza, J., Generelo, E., y Julián, J.A. (2010). Comportamientos sedentarios y patrones de actividad física en adolescentes. Revista Internacional de Medicina y Ciencias de la Actividad Física y del Deporte, 40, 410-427.

Ajzen, I. (1991). The theory of planned behavior. Organizational behavior and human decision processes, 50, 179-211.

American Psychological Association (2002). Ethical principles of psychologists and code of conduct (Amended June 2, 2010). Tomado de http://www.apa.org/ethics/code/

Ames, C. (1992). Classrooms: goals, structures, and student motivation. Journal of Educational Psychology, 84(3), 261-271.

Baena-Extremera, A., Granero-Gallegos, A., Sánchez-Fuentes, J. A., y Martínez-Molina, M. (2013). Apoyo a la autonomía en Educación Física: antecedentes, diseño, metodología y análisis de la relacción con la motivación en estudiantes adolescentes. Retos. Nuevas tendencias en Educación Física, Deporte y Recreación, 24, 46-49.

Bauman, A., y Merom, D. (2002). Measurement and surveillance of physical activity in Australia - an introductory guide. Australasian Epidemiologist, 9(2), 2-6.

Belando, N. (2013). Motivación autodeterminada y compromiso deportivo en estudiantes adolescentes. Universidad Miguel Hernández deElche, Alicante.

Carazo-Vargas, P., y Moncada-Jiménez, J. (2015). A meta-analysis on the effects of exercise training on the VO2 max in children and adolescents. Retos. Nuevas tendencias en Educación Física, Deporte y Recreación, 27, 184-187.

Castillo, I., y Moncada, J. (2012). El efecto de la frecuencia de participación en un programa de ejercicios contra resistencia sobre la estima y la satisfacción corporal de mujeres universitarias costarricenses. Revista Iberoamericana de Psicología del Ejercicio y el Deporte, 5(2), 195-212.

Cervelló, E., Moreno, J. A., Alonso, N., y Iglesias, D. (2006). Goal orientation, motivational climate, and dispositional flow of high school students engaged in exrtacurricular physical activity. Perceptual \& Motor Skills, 102, 87-92. DOI: 10.2466/ PMS.102.1.87-92

Christodoulidis, T., Papaioannou,A., y Digelidis, N. (2001). Motivational climate and attitudes towards exercise in greek senior high school: a year-long intervention. European Journal of Sport Science, 1(4).

Cohen, J. (1988). Statistical power analysis for the behavioral sciences. Hilsdale, New Jersey: LEA.

Comisión Europea/EACEA/Eurydice. (2013). La educación física y el deporte en los centros escolares de Europa. Informe Eurydice. Luxemburgo: Oficina de Publicaciones de la Unión Europea.

Coterón, J., Sampedro, J., Franco, E., Pérez-Tejero, J., y Refoyo, I. (2013). The role of basic psychological needs in predicting dispositional flow of basketball players in training. Differences by sex. Revista de Psicología del Deporte, 22(1), 187-190.

Csikszentmihalyi, M. (1975). Beyond boredome and anxiety. San Francisco: Jossey-Bass.

Csikszentmihalyi, M., Abuhamdeh, S., y Nakamura, J. (2005). Flow. En A. J. Elliot y C. Dweck (Eds.), Handbook of competence and motivation (pp. 598-608). New York: Guilford Publications.

Currie, C., Gabhainn, S. N., y Godeau, E. (2008). Inequalities in young people's health. Health behaviour in school-aged children. (pp. 105-112). Copenhagen, Denmark: WHO Regional Office for Europe.

Deci, E. L., y Ryan, R. M. (1985). Intrinsic motivation and self- determination in human behaviour. New York: Plenum.

Duda, J. L., y Allison, M. T. (1990). Cross-cultural analysis in exerecise and sport psychology: a void in the field. Journal of Sport and Exercise Phychology, 12, 114-131.

García Sandoval, J. R. (2013). La motivación en la práctica de ejercicio físico en adolescentes mexicanos y españoles. Universidad de Sevilla, Sevilla.

Godin, G. y Shephard, R. J. (1985). A simple method to assess exercise behavior in the community. Canadian Journal of Applied Sport Sciences, 10, 141-146.

Gomes, L. P., Tadeu, M., Cordeiro, W., Stabelini, A., Chacón-Araya, Y., y de Campos, W. (2015). Aerobic power in prepubescent children with different levels of physical activity. Retos. Nuevas tendencias en Educación Física, Deporte y Recreación, 27, 203-205.

González-Cutre, D. (2009). Motivación, creencias implícitas de habilidad, competencia percibida y flow disposicional en clases de educación física. Universidad de Almería.

Goudas, M., Biddle, S., y Fox, K. (1994a). Achievement Goal Orientations and Intrinsic Motivation in Physical Fitness Testing With Children. Pediatric Exercise Science, 6(2), 159-167.

Goudas, M., Biddle, S., y Fox, K. (1994b). Perceived locus of causality, goal orientations, and perceived competence in school physical education classes. British Journal of Educational Psychology, 64, 453-463.

Granero-Gallegos, A., y Baena-Extremera, A. (2014). Predicción de la motivación autodeterminada según las orientaciones de meta y el clima motivacional en Educación Física. Retos. Nuevas tendencias en Educación Física, Deporte y Recreación, 25, 23-27.

Granero-Gallegos, A., Baena-Extremera, A., Pérez-Quero, F. J., OrtizCamacho, M. M., y Bracho-Amador, C. (2014). Validación española del «intention to partake in leisure-time physical activity». Retos. Nuevas tendencias en Educación Física, Deportey Recreación, 26, 40-54.

Hagger, M., Chatzisarantis, N., y Biddle, S. (2002). The influence of autonomous and controlling motives on physical activity intentions within the Theory of Planned Behaviour. British Journal of Health Psychology, 7, 283-297.

Hein, V., Müür, M., y Koka, A. (2004). Intention to be physically active after school graduation and its relationship to three types of intrinsic motivation. European Physical Education Review, 10(1), 5-19.

Isorna, M., Rial, A., y Vaquero-Cristóbal, R. (2014). Motivaciones para la práctica deportiva en escolares federados y no federados. Retos. Nuevas tendencias en Educación Física, Deporte y Recreación, 25, 80-84.

Jackson, S. A. (1996). Toward a conceptual understanding of the flow experience in elite athletes. Research Quarterly for Exercise y Sport, 67(1), 76-90.

Jackson, S. A., y Eklund, R. C. (2002). Assessing Flow in Physical Activity: The Flow State Scale-2 and Dispositional Flow Scale-2. Journal of Sporty Exercise Psychology, 4, 133-150.

Jackson, S. A., y Marsh, H. W. (1996). Development and Validation of a Scale to Measure Optimal Experience: The Flow State Scale. Journal of Sport \& Exercise Psychology, 18, 17-35.

Karageorghis, C. I., Vlachopoulos, S., y Terry, P. C. (2000). Latent variable modelling of the relationship between flow and exerciseinduced feelings: an intuitive appraisal perspective. European Physical Education Review, 6(3), 230-248.

Kimiecik, J. C. (2000). Learn to love exercise. Psychology Today, 33(1), 20-22.

Lintunen, T., Valkonen, A., Leskinen, E., y Biddle, S. (1999). Predicting physical activity intentions using a goal perspectives approach: a study of Finnish youth. Scandinavian Journal of Educational Research, 9, 344-352.

López-Walle, J., Tomás, I., Castillo, I., Tristán, J., y Balaguer, I. (2011). Invarianza factorial del TEOSQ en jóvenes deportistas mexicanos y españoles. Revista Mexicana de Psicología, 28, 53-61. 
Mandigo, J. L., y Thompson, L. (1998). Go with their flow: How flow theory can help practitioners to intrinsically motivate children to be physically active. Physical Educator, 55, 145-159.

Moreno-González, R. (2014). Relación entre las metas de logro, la motivación autodeterminada, las creencias implícitas de habilidad y el autoconcepto físico en educación física. Universidad Miguel Hernández de Elche, Alicante.

Moreno, J.A., Cervelló, E., Martínez-Galindo, C., y Alonso, N. (2007). Predicción del flow disposicional según el clima motivacional y el trato generado por el profesor en clase de educación física. Análisis y Modificación de la Conducta(33), 207 - 228.

Moreno, J. A., González-Cutre, D., y Chillón, M. (2009). Preliminary validation in Spanish of a scale designed to measure motivation in physical education classes: The perceived locus of causality (PLOC) scale. The Spanish Journal of Psychology, 12(1), 327-337.

Moreno, J. A., Moreno, R., y Cervelló, E. (2007). El autoconcepto físico como predictor de la intención de ser físicamente activo. Psicología y Salud, 17(2), 261 - 267.

Nicholls, J.G. (1989). The competitive ethos and democratic. Cambridge: Harvard University Press.

Nishida, T. (1991). Achievement motivation for learning in physical education class: A cross cultural study in four countries. Perceptual and Motor Skills, 72, 1183 - 1186.

Oviedo, G., Sánchez, J., Castro, R., Calvo, M., Sevilla, J. C., Iglesias, A., y Guerra, M. (2013). Niveles de actividad física en población adolescente: estudio de caso. Retos. Nuevas tendencias en Educación Física, Deporte y Recreación, 23, 43-47.

Peiró, C., y Sanchis, J. R. (2004). Las propiedades psicométricas de la versión inicial del cuestionario de orientación a la tarea y al ego (TEOSQ) adaptado a la educación física en su traducción al castellano. Revista de Psicología del Deporte, 13(1), 25-39.

Pulido, J. J., Sánchez-Oliva, D., Amado, D., González-Ponce, I., y Sánchez-Miguel, P.A. (2014). Influence of motivational processes on enjoyment, boredom and intention to persist in young sportspersons. South African Journal for Research in Sport, Physical Education y Recreation (SAJR SPER), 36(3), 135-149. efecto: ¿Un nuevo canon para la investigación? Onomázein, 16(2), 159-170.

Ruiz, L. M., Graupera, J. L., Contreras, O., y Nishida, T. (2004). Motivación de logro en educación física escolar: un estudio comparativo entre cinco países. Revista de Educación, 333, 345-361.

Ruiz-Juan, F., y Baena-Extremera, A. (2015). Predicción de las metas de logro en educación física a partir de la satisfacción, la motivación y las creencias de éxito en el deporte. Revista Iberoamericana de Psicología del Ejercicio y el Deporte, 10(2), 193-203.
Quezada, C. (2007). Potencia estadística, sensibilidad y tamaño del

Shephard, R. J., y Trudeau, F. (2000). The legacy of physical education: influences on adult lifestyle. Pediatric Exercise Science, 12(1), 3450.

Skjesol, K., y Halvari, H. (2005). Motivational climate, achievement goals, perceived sport competence, and involvement in physical activity: Structural and mediator models. Perceptual \& Motor Skills, 100(2), 497-523. DOI: 10.2466/PMS.100.2.497-523.

Sproule, J., Wang, C. K. J., Morgan, K., McNeill, M., y McMorris, T. (2007). Effects of motivational climate in Singaporean physical education lessons on intrinsic motivation and physical activity intention. Personal and Individual Differences, 43, 1037-1049. DOI: 10.1016/j.paid.2007.02.017.

Taylor, I. M., Ntoumanis, N., Standage, M., y Spray, C. M. (2010). Motivational predictors of physical education students' effort, exercise intentions, and leisure-time physical activity: a multilevel linear growth analysis. Journal of Sport and Exercise Psychology, 32, 99-120.

UNESCO. (2015). Educación Física de Calidad. Guía para los Responsables Políticos. París: Organización de las Naciones Unidas para la Educación, la Ciencia y la Cultura.

Velázquez, R., Hernández, J. L., Garoz, I., y Martínez, M. E. (2015). Auto-eficacia motriz, educación física y actividad física en adolescentes brasileños y españoles. Revista Internacional de Medicina y Ciencias de la Actividad Física y del Deporte, 15(60), 632-645. DOI: 10.15366/rimcafd2015.60.002.

Vlachopoulos, S., Asci, F. H., Cid, L., Ersoz, G., González-Cutre, D., Moreno, J. A., y Moutao, J. (2013). Cross-cultural invariance of the basic psychological needs in exercise scale and need satisfaction latent mean differences among Greek, Spanish, Portuguese and Turkish samples. Psychology of Sport y Exercise, 14, 622-631. DOI: 10.1016/j.psychsport.2013.03.002

Walling, M. D., y Duda, J. L. (1995). Goals and their associations with beliefs about success in and perceptions of the purposes of physical education. Journal of Teaching in Physical Education, 14(2), 140156.

Wang, C. K. J., Hagger, M., y Liu, W. C. (2009). A cross-cultural validation of perceived locus of causality scale in physical education context. Research Quarterly for Exercise and Sport, 80(2), 313 325.

Wang, C. K. J., Liu, W. C., Biddle, S., y Spray, C. (2005). Cross-cultural validation of the conceptions of the nature of athletic ability questionnaire version 2. Personal and Individual Differences, 38, 1245-1256. DOI: 10.1016/j.paid.2004.08.007

Xiang, P., Lee, A., y Shen, J. (2001). Conceptions of ability and achievement goals in physical education: Comparisons of American and Chinese students. Contemporary Educational Psychology, 26, 348-365.

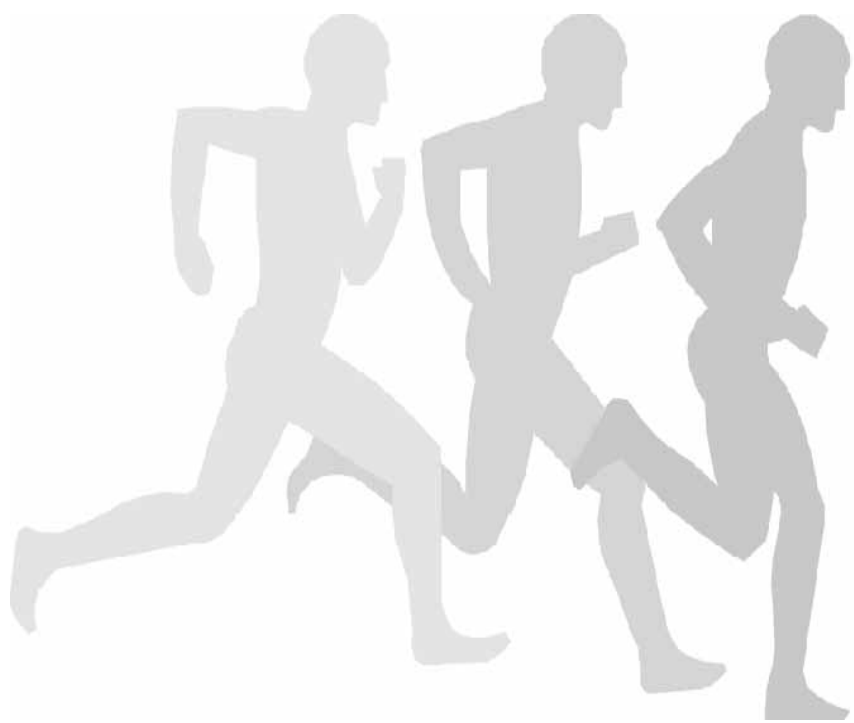

